\title{
Combination of Fascia Iliaca Compartment Block on the Surgical Side with Sacral Spinal Anesthesia for Hip to Knee Surgery
}

\author{
Radhashyam Paria ${ }^{1}$, Smarajit Surroy ${ }^{1}$, Mousumi Majumder ${ }^{1}$, Baishakhi Paria ${ }^{2}$, \\ Soma Sengupta ${ }^{3}$, GoutamDas, ${ }^{4}$ Anshuman Paria ${ }^{5}$ \\ 1(Dept of Anesthesiology, Howrah Orthopedic Hospital, Eastern Railways, WB) \\ 2(Department of Community Medicine, National Medical College) \\ 3(Dept of Anesthesiology, SSKM hospital, WB) \\ 4(Department of Pediatric Medicine, North Bengal Medical College, West Bengal) \\ 5(Dept of Neonatology, SSKM hospital, WB)
}

\begin{abstract}
Background and Aims: Different kinds of adjutants are used with local anesthetics during intrathecal procedure for prolongation of action. However, they are not completely free from side effects like postoperative hypoxia and cardiovascular instability. But subsequently, the use of preoperative femoral nerve block on surgical side provides beneficial analgesia to relieve fracture pain before operation, to make cooperative during administration of spinal anesthesia, to act as adjutants to intrathecal procedure and lastly to provide postoperative analgesia without side effect like central depression.

Method: 50 (fifty) patients were administered fascia iliac compartment block (FICB) on the surgical side before intrathecal procedure with $30 \mathrm{ml}$ of $0.5 \%$ injection ropivacaine. Sacral spinal anesthesia was administered through the first or second dorsal foramen of sacrum with $3 \mathrm{ml}$ of $0.5 \%$ injection bupivacaine. Periodically, the estimation of blood pressure, heart rate and oxygen concentration was carried out.

Results: 50 (fifty) patients were administered FICB successfully on surgical side preoperatively. Pain at the fractured site was relieved completely and all patients cooperated at the time of intrathecal procedure. Hemodynamic fluctuation was absent in operative period. Prolonged analgesia was noticed in postoperative period.

Conclusion: High quality of analgesia, delivered by FICB is appropriate to relieve the pain in preoperative period from fractured site, to act as an adjutant to sacral spinal anesthesia during surgery and to provide prolonged analgesia after surgery without adverse reaction like hypoxia and central depression to combat surgical stress response.
\end{abstract}

\section{Introduction}

In the current era of highly developed modern technological implication in both surgery and anesthesia, motivation of anesthesiologists in the line of regional nerve block technique became a most welcome move in view of avoidance of central depression with provision of high quality analgesia immediate after surgery to guard against surgical stress response. Such move has been celebrated with introduction of combined use of central and specific peripheral nerve block technique joint together to enforce their beneficial effects with minimizing their drawbacks. Unpredictable and fixed durable spinal anesthesia frequently needs intrathecal adjutants like opioid [1 ] or clonidine [2-4 ] or fentanyl 5-8 ] or morphine [9--12] to combat the deficiency of intrathecal procedure, even after knowing their individual complications of central depression and postoperative hypoxia particularly in elderly patients.

Excruciating pain at fracture site and its exaggeration on movement during transport and intrathecal procedure are the main culprits for increasing stress, strain and mental agony of the patients. Profound analgesia is the essential component of anesthesia to control surgical stress response both during and after surgery. In relation to pre-operative medication, the usual use of systemic opioid frequently relieves pain with a lot of side effects particularly in the older group of patients. However, in search of good analgesic without side effects, we hypothesized that fascia iliaca compartment block (FICB) may be perfectly eligible for the above purpose in case of femur fracture. Based on such assumption, we designed our study to administer FICB on fracture side of femur pre-operatively to evaluate its efficacy and effectiveness as preoperative, intra-operative and postoperative analgesia.

\section{Methods}

With written informed consent for procedure and study, we recruited 50 patients, aged between 60 to 90 years, with ASA physical status class II and III, undergoing femur surgery in the period of March 2013 to November 2013. At the time of preoperative assessment to eliminate the cases, contraindicated for spinal 
anesthesia, we examined the area surrounding the inguinal crease on the fracture side to exclude the local infection for further procedure like FICB.

In the operation room, after establishment of intravenous infusion and identification of anatomical landmarks like anterior superior iliac spine and pubic tubercle on the fractured side in the supine position of the patient we adopted aseptic preparation of the part. We located a point of $1 \mathrm{~cm}$ below from the junction of lateral one-third and medial two-third of the inguinal ligament and felt the pulsation of femoral artery. We inserted an intramuscular needle of $21 \mathrm{G}$ perpendicularly to the skin and advanced until we felt a loss of resistance as first click offered by fascia lata. With further progress of the needle, we experienced another loss of resistance as a second click offered by fascia iliaca and injected $30 \mathrm{ml}$ of $0.5 \%$ injection ropivacaine. However, at the time of administration of the drug, we adopted precaution to avoid the intravascular injection by guarding the femoral artery and displacing medially by finger of the hand. With subsiding pain at the fractured site after administration of this block, we completed intrathecal procedure as usual in sitting position of the patient but without pain.

After completion of femoral nerve block, anatomical landmarks of dorsal foramens of sacrum were identified. We identified the spinous process of fourth lumbar vertebra (L4) and tip of coccyx. Next, we located the mid-point of above two landmarks as third sacral vertebra (S3). The first sacral vertebra (S1) was located as mid-point of L4 and S3. The second sacral vertebra (S2) was found at the mid-point between S1 and S3. The spinous processes of $\mathrm{S} 1$ and $\mathrm{S} 2$ are found approximately $2.5 \mathrm{~cm}$ apart and their respective foramina lie $1.5 \mathrm{~cm}$ caudal and lateral to them. Similarly S3 foramina lie $1.5 \mathrm{~cm}$ caudal and lateral to S3 vertebra.

The above described land marks were further confirmed by visualizing their relations to other structures. L4 is on the line joining the iliac crests. The tip of coccyx lies on the line joining the femoral acetabula. The spinous process of S2 lie at the level of posterior superior iliac spine. It is often detected by a dimple of skin. The $\mathrm{S} 3$ lie at the level of posterior inferior iliac spine.

We infiltrated the identified area with $6 \mathrm{ml}$ of $1 \%$ xylocaine and administered sacral spinal anesthesia $[13,14,15]$ through $2^{\text {nd }}$ dorsal foramen of sacrum with intrathecal injection of $3 \mathrm{ml}$ of $0.5 \%$ injection bupivacaine. The intrathecal injection was administered after noticing clear flow of cerebrospinal fluid [CSF] to avoid intravascular injection.

We estimated the onset of sensory or motor block(interval between spinal and loss of sensation or motor function of lower limbs respectively) and duration of sensory or motor block(interval between spinal and recovery of sensation or motor respectively) and presented all collected data for statistical analysis.

Prior to the anesthetic procedure, we monitored every patient for non-invasive blood pressure, electrocardiogram, heart rate, and oxygen concentration and repeated at every 5 minutes. Intra-operative hemodynamic status and post-operative analgesic period were the main target to note for statistical analysis.

\section{Results}

We administered FICB to 50 patients before intrathecal injection of bupivacaine to get rid of pain from fracture site. Within 5 minutes interval, pain reduced to minimal intensity and patients became cooperative for intrathecal procedure in sitting position. During intra-operative period, hemodynamic stability was noted both in blood pressure and heart rate. Hemodynamic profile in table no 1 indicated such. Patients did not require supplementary analgesia in intravenous route. They did not need sedation to relieve anxiety. The motor and sensory block developed within short time. Post-operative analgesia lasted for 15 hours. Minimal amount of analgesic needed in the last part of post-operative period. Sensory block extended to 12th thoracic dermatome. The result of sensory and motor nerve block profile is cited in table no 1 . The outcome of all patients was smooth. All elderly patients of average height and weight were participants of this study. Their demographic profile is cited in the table no. 1.

\section{Discussion}

Fracture pain is severe in nature, exaggerated on movement causing tremendous mental agony. For the relief of such excruciating pain at the fracture site, traditionally, we use systemic opioid, although it is not free from side effects particularly in elderly patients. Other options to relieve such unbearable pain are less effective to comfort the patients. Analgesic effect of FICB is sufficient and superior at all time.

FICB is a method of peripheral nerve block for femoral, lateral cutaneous and obturator nerves and is responsible to offer analgesia for the area of the femur, hip and knee joint. It is a simple procedure, technically easy to perform and highly effective to provide analgesia before surgery as pre-operative medication for painless transport and intrathecal procedure. Its analgesic effect as an adjutant to spinal anesthesia in intra-operative period is more accountable and preferable than systemic opioid and even combined effect with epidural. Its analgesic effect to control surgical stress response during and after operative procedure in relation to the femur, hip and knee joint magnificently claims superiority in managing the deficits presented by spinal anesthesia. 
The consideration of the anatomical configuration of the fascia iliaca (FI) covering the femoral nerve and its branches reveals that FI compartment is a potential space between FI and iliacas muscle itself. FI is located anterior to iliacas muscle within pelvis. The femoral nerve and lateral cutaneous nerve lie under this fascia whereas obturator nerve deep ilicas muscle. The local anesthetic solution, deposited in this compartment beneath the FI should spread between this fascia and muscle. Femoral and lateral cutaneous nerves are involved quickly depending on volume of local anesthetic. The obturator nerve is not usually involved by FICB although this is not so important in pain relief of hip and knee. All the above mentioned nerves belong to lumbar plexus.

FICB provides analgesia of high quality.[17-19]The analgesic property of FICB is superior to systemic oipoid [20,21]but inferior to epidural analgesia with fewer complications[22] Overall it is a very low profile risky procedure to block the femoral, obturator and lateral cutaneous nerves. The risk of intravascular injection, toxicity of local anesthetic and mechanical nerve damage is extremely low. The chance of infection is rare with good aseptic preparation of the site. Large volume of local anesthetic needed for FIRB ensures good spread and reduces the risk of failure, however, the chance of drug toxicity is always present. So closed observation with monitoring at least for the first 15 minutes is essential and mandatory.

Generally sacral spinal anesthesia does not act as hypotension producing agent as lumbar or lower thoracic spinal anesthesia. Restricted cephalic extension of local anesthetic in case of sacral spinal anesthesia leads to less involvement of sympathetic outflow of the spinal cord at the thoracic region. Only unidirectional cephalic intrathecal spread of local anesthetic is possible as local anesthetic is deposited at the bottom of dural sac. Caudal spread of local anesthetic is absent in sacral spinal anesthesia. So, cephalic spread of local anesthetic is accompanied with decreasing the drug concentration due to dilution in lumbosacral cerebrospinal fluid (CSF) and distribution of drugs to neural tissues. This explains the less involvement of sympathetic outflow by local anesthetic.

The most unfavorable and undesirable fixed duration of anesthesia, contributed by intrathecal procedure needs adjutants like opioid [1] or clonidine [2-4 ] or fentanyl [5-8 ] or morphine [9-12] for prolongation of action, although this attempt is complicated with central depression and postoperative hypoxia particularly in elderly patients.

Most of the patients did not require systemic opioid at least for 15 hours in the postoperative period and when they required, they needed minimum dose. The preoperative administration of FICB not only prolonged the postoperative analgesia, but also reduced further dose of systemic analgesic. The hemodynamic stability in peri-operative period increases its further importance in clinical practice.

\section{Conclusion}

The analgesic property of FICB in case of femur surgeries is useful as pre-operative medication, adjutant to spinal anesthesia and post-operative analgesic to control surgical stress response particularly in elderly patients.

\section{Reference}

[1]. Popping DM, Elia N, Marret E, Wenk M and Tramer MR. Opioids added to local anesthetics for single-shot intrathecal anesthesia in patients undergoing minor surgery: a meta-analysis of randomized trials. Pain.2012; 153:784-93.

[2]. Sethi BS, Samuel M, Sreevastava D. Efficacy of analgesic effects of low dose intrathecal clonidine as adjuvant to bupivacaine. Indian J Anaesth 2007;51:415-9.

[3]. Grandhe RP, Wig J, Yaddanapudi LN. Evaluation of bupivacaine-clonidine combination for unilateral spinal anesthesia in lower limb orthopaedic surgery. J Anaesth Clin Pharmacol 2008;24:155-8.

[4]. Niemi L. Effects of intrathecal clonidine on duration of bupivacaine spinal anaesthesia, hemodynamic and postoperative analge sia in patients undergoing knee arthroscopy. Acta Anesthesiol Scand 1994;38:724-8.

[5]. Dahlgren G, Hultstrand C, Jakobsson J et al. Intrathecal sufentanil, fentanyl or plaecebo added to bupivacaine for caesarean section. Anesth Analg 1997; 85; 1288-93.

[6]. Manullang TR, Viscomi CM, Pace NL. Intrathecal fentanyl is superior to intravenous ondansetron for prevention of perioperative nausea during caesarean delivery with spinal anaesthesia. Anesth Analg 2000; 90: 1162-6.

[7]. Shendi D, Cooper GM, Bowden MI. The influence of IT fentanyl on the characteristics of subarchnoid block for Caesarean section. Anaesthesia 1998; Jul 53(7): 706-10.

[8]. Chu CC, Shu SS, Lin SM et al. The effect of intrathecal bupivacaine with combined fentanyl in caesarean section. Acta Anaesthesiol Sin 1995; Sep 33(3): 149-54

[9]. Bailey PL, Rhondeau S, Schafer PG, et al Dose-response pharmacology of intrathecal morphine in human volunteers. Anesthesiology 1993;79:49-59.

[10]. Beaussier M, Weickmans H, Parc Y, et al Postoperative analgesia and recovery course after major colorectal surgery in elderly patients: a randomized comparison between intrathecal morphine and intravenous PCA morphine. Reg Anesth Pain Med2006;31:531-8.

[11]. Blay M, Orban JC, Rami L, et al Efficacy of low-dose intrathecal morphine for postoperative analgesia after abdominal aortic surgery: a double-blind randomized study. Reg Anesth Pain Med 2006;31:127-33.

[12]. Block BM, Liu SS, Rowlingson AJ, Cowan AR, Cowan JA Jr, Wu CL Efficacy of postoperative epidural analgesia: a metaanalysis. J Am Med Assoc2003;290:2455-63.

[13]. Paria R, Surroy S, Majumder M, Paria A, Paria B, Das G. Sacral spinal anesthesia. Indian J Anesth. 2014; 58(1) : 80-2

[14]. Paria R, Surroy S, Majumder M, Paria B, Sengupta S, Paria A. Sacral Saddle Block IOSR- JDMS 2014; 13(4) 39-40.

[15]. Paria R, Surroy S, Majumder M, Paria B, Sengupta S, Paria A. Sacral Epidural Anesthesia IOSR-JD MS 2014; 13(5) 10-11 
[16]. Volka, J,D,. Hadzic,A,. Drobnik, 1,.Ernest,A,.Reiss,W,. Thys,D,M. Anatomical landmarks for femoral nerve block: A comparison of four needle insertion sites. Anesth. Analg. 89;1467:1999

[17]. Dahl JB, Christiansen CL, Daugaard JJ, et al. Continuous blockade of the lumbar plexus after knee surgery: Postoperative analgesia and bupivacaine plasma concentrations. Anaesthesia 1988;43:1015-8.

[18]. Edwards E, Wright M. Continuous low dose 3-in-1 nerve blockade for postoperative pain relief after total knee replacement. Anesth Analg 1992;75:265-7.

[19]. Matheny JM, Hanks GA, Rung GW, et al. Comparison of patient-controlled analgesia and continuous lumbar plexus block after anterior cruciate ligament reconstruction. Arthroscopy 1993;9:87-90.

[20]. Serpell MG, Millar FA, Thomson MF. Comparison of lumbar plexus block versus conventional opioid analgesia after total knee replacement. Anaesthesia 1991;46:275

[21]. Schultz P, Anker-Moller E, Dahl JB, et al. Postoperative pain treatment after open knee surgery: continuous lumbar plexus block with bupivacaine versus epidural morphine. Reg Anesth 1991;16:34-7

[22]. Mulroy, M.F, Larkin, K.L., Batra, M.S., Hodgson, P.S., Owens, B.D., Femoral nerve block with $0.25 \%$ or $0.5 \%$ bupivacaine improves postoperative analgesia following outpatient arthroscopic anterior cruciate ligament repair. Reg. Anesth. Pain Med. 26; $24: 2001$

Table 1 : Showing demographic profile, hemodynamic profile and analgesic profile of patients. DEMOGRAPHIC PROFILE

Age (years)

$68.38 \pm 8.08$

Height $(\mathrm{Cm})$

$157.96 \pm 4.67$

Weight $(\mathrm{Kg})$

$55.04 \pm 3.02$

$\operatorname{Sex}(\mathrm{M}: \mathrm{F})$

$28: 22$

ODYNAMIC PROFILE

$112.32 \pm 7.62$

Systolic blood pressure $(\mathrm{mmHg})$

$89.92 \pm 12.75$

Heart beats (beats/min)

$98.94 \pm 0.89$

ANALGESIC PROFILE

Postoperative analgesic(hours)

$15.82 \pm 2.34$ 